\title{
Making sense of the CT Urogram
}

\author{
Hiram Shaish ${ }^{1}$
}

Received: 29 October 2019 / Accepted: 8 November 2019 / Published online: 17 January 2020

(C) European Society of Radiology 2019

\section{Key Points}

- There is great variation in the CT urogram protocol throughout the literature.

- The study by Renard-Penna et al provides a useful rigorous expert consensus on CT urography technique.

- The study by Rud et al suggests that the excretory phase may not be as necessary as previously thought for visualizing upper tract urothelial carcinoma.

The CT urogram (CTU) has evolved into a cornerstone of urologic disease diagnosis and management, performed for a myriad of diseases, both benign and malignant. Since the original head to head comparison between the excretory phase at CT with excretory urography (intravenous pyelography or IVP) for opacification of the collecting systems, reported by McNicholas et al in 1998 [1], radiologists have endeavored to improve CTU technique for evaluating the kidneys and urinary tracts. Studies have examined the utility of multiple postcontrast phases, timing of the excretory phase, administration of diuretics, administration of spasmolytic agents, patient hydration, abdominal compression, and different patient positions $[2,3]$. The results of these interventions have resulted in a vast number of often technically cumbersome and/or high radiation dose CTU protocols [4], ultimately being sorted out at the local level based on practice preferences and experience rather than expert consensus guidelines which are lacking.

The study by Renard-Penna et al [5] brings some semblance of order to the chaos of CTU techniques. The authors conducted a rigorous Delphi consensus conference on the topic of CTU technique. Forty-two expert uroradiologists from the French Society of Genitourinary Imaging reached consensus on $96(70 \%)$ of the initial 138 statements. Of note, the administration of $20 \mathrm{mg}$ of furosemide before intravenous contrast injection was deemed necessary and the optimal delay for the excretory phase was determined to be 7 minutes. Other

This comment refers to the articles available at https://doi.org/10.1007/ s00330-019-06521-0 and https://doi.org/10.1007/s00330-019-06529-6.

Hiram Shaish

Hs2926@cumc.columbia.edu

1 Columbia University Medical Center, 630 West 168th St, New York, NY 10032, USA maneuvers for distending the collecting systems including oral or intravenous hydration were not deemed useful. The optimal combination of post-contrast phases should be tailored to specific patient populations and clinical scenarios.

In the specific clinical setting of hematuria in an older adult with risk factors for urothelial carcinoma, diuresis is vital as it both distends the collecting systems and dilutes the excreted contrast. In this manner, there is improved visualization of the mid to distal ureters and small urothelial carcinomas can be seen more easily as filling defects in a sea of excreted dilute contrast. An additional unproven benefit of diuresis is more homogenous opacification of a distended urinary bladder which may help better visualize bladder tumors as well [6].

Importantly, the authors note that small and/or sessile tumors may not produce filling defects on the excretory phase and therefore, the corticomedullary or nephrographic phases should also be used. This point has been a focus of recent investigation. First, as a way of background, the traditional focus on the excretory phase is likely a remnant of the excretory urography days. Before the era of CT, the only reliable way to visualize upper tract tumors radiologically was to demonstrate filling defects and/or obstruction on excretory urography. Thus, the initial focus of CTU research was on reproducing noninferior excretory phase images. Nevertheless, as alluded to by Renard-Penna et al, the excretory phase is not the only way to detect these tumors. Recent studies have shown that urothelial tumors avidly enhance and in fact may sometimes be better seen with an earlier phase of imaging on a background of normal urothelium and nonopacified urine [7-10]. Importantly, diuresis is potentially equally important on these non-excretory phases as it distends the collecting systems with nonopacified urine thereby allowing for better visualization of the urothelium itself. 
In this regard, the study by Rud et al [11] calls into question the dogmatic focus on the excretory phase, thus supporting the overarching goal of Renard-Penna et al to simplify CT evaluation of the kidneys and collecting systems. Rud et al retrospectively reviewed the nephrographic and excretory phases from CT urograms in patients with proven upper tract urothelial carcinoma. They report that all seven of the upper tract tumors were visible to both blinded readers on the nephrographic phase. This study, as well as several prior studies [7-10], begin to dispel the notion that the excretory phase is the only phase for detection of bladder and upper tract urothelial tumors. Rather, an earlier post-contrast phase may be sufficient and at the very least should be used with the excretory phase in a complementary fashion, corroborating each other as well as improving sensitivity and specificity, particularly of small and/or sessile lesions.

On this last point, it is important to circle back to the splitbolus technique. Renard-Penna et al state that split-bolus should be favored whenever possible to decrease dose. While this may be warranted in younger patients who have potentially increased risk from radiation exposure and decreased incidence of malignant disease, the same cannot be said of older patients with risk factors for urothelial carcinoma in whom the risk-benefit ratio is quite different. The splitbolus CTU eliminates the possibility of visualizing the hyperenhancement of urothelial tumors on a background of nonopacified urine as well as potentially masking the diluted excreted contrast (in the case of diuresis) in the ureters by the adjacent enhanced vessels. Furthermore, combining a diuretic with the split-bolus technique has not been reported on and, at least anecdotally, may result in "splitting the baby" in terms of optimizing the nephrographic and excretory phases. Although we reported only a small number of missed upper tract urothelial carcinomas in the largest split-bolus cohort in the literature [12], this may have been primarily due to the low incidence of upper tract urothelial tumors in general, and not necessarily testament to the adequacy of the split-bolus technique. Additionally, we reported a low sensitivity of 55\% for the detection of bladder tumors. In fact, the split-bolus technique has not been shown to suffice for evaluation of the bladder.

In summary, the studies by Renard-Penna et al and Rud et al $[5,11]$ provide critical first steps towards much needed clarity in understanding how best to perform CT urography as well as insight and perspective on the role of the excretory phase in the detection of urothelial carcinoma. These important publications will undoubtedly lead to further global consensus as well as larger studies to elucidate the utility of the different CTU phases.

Acknowledgments Lawrence Schwartz, MD.

Funding information The authors state that this work has not received any funding.

\section{Compliance with ethical standards}

Guarantor The scientific guarantor of this publication is Lawrence Schwartz MD.

Conflict of Interest The authors of this manuscript declare no relationships with any companies, whose products or services may be related to the subject matter of the article.

Statistics and biometry No complex statistical methods were necessary for this paper.

Informed consent Not required (editorial)

Ethical approval Institutional Review Board approval was not required because this is an editorial.

\section{Methodology}

- Editorial

\section{References}

1. McNicholas MM, Raptopoulos VD, Schwartz RK et al (1998) Excretory phase CT urography for opacification of the urinary collecting system. AJR Am J Roentgenol 170:1261-1267

2. Nolte-Ernsting C, Cowan N (2006) Understanding multislice CT urography techniques: many roads lead to Rome. Eur Radiol 16: 2670-2686

3. Sanyal R, Deshmukh A, Singh Sheorain V, Taori K (2007) CT urography: a comparison of strategies for upper urinary tract opacification. Eur Radiol 17:1262-1266

4. Yecies T, Bandari J, Fam M, Macleod L, Jacobs B, Davies B (2018) Risk of radiation from computerized tomography urography in the evaluation of asymptomatic microscopic hematuria. J Urol 200:967-972

5. Renard-Penna R, Rocher L, Roy C et al (2019) Imaging protocols for CT urography: results of a consensus conference from the French Society of Genitourinary Imaging. Eur Radiol. https://doi. org/10.1007/s00330-019-06529-6

6. Trinh TW, Glazer DI, Sadow CA, Sahni VA, Geller NL, Silverman SG (2018) Bladder cancer diagnosis with CT urography: test characteristics and reasons for false-positive and false-negative results. Abdom Radiol (NY) 43:663-671

7. Kupershmidt M, Margolis M, Jang HJ, Massey C, Metser U (2011) Evaluation of upper urinary tract tumors with portal venous phase MDCT: a case-control study. AJR Am J Roentgenol 197:424-428

8. Metser U, Goldstein MA, Chawla TP, Fleshner NE, Jacks LM, O'Malley ME (2012) Detection of urothelial tumors: comparison of urothelial phase with excretory phase CT urography-a prospective study. Radiology 264:110-118

9. Park JJ, Park BK, Kim CK (2016) Single-phase DECT with VNCT compared with three-phase CTU in patients with haematuria. Eur Radiol 26:3550-3557

10. Helenius M, Dahlman P, Lonnemark M, Brekkan E, Wernroth L, Magnusson A (2016) Comparison of post contrast CT urography phases in bladder cancer detection. Eur Radiol 26:585-591

11. Rud E, Galtung KF, Lauritzen PM et al (2019) Examining the upper urinary tract in patients with hematuria - time to revise the CT urography protocol? Eur Radiol. https://doi.org/10.1007/s00330-019-06521-0

12. Shaish H, Newhouse JH (2017) Split-bolus CT urogram: Is less more? Abdom Radiol (NY) 42:2119-2126

Publisher's note Springer Nature remains neutral with regard to jurisdictional claims in published maps and institutional affiliations. 